\title{
Benefits of Understanding Systemic Racism in Forming Clinician-Patient Relationships to Reduce Black Infant Mortality
}

\author{
Michelle A. Gotto'; Laura Morello²; Marsha Michie ${ }^{2}$ \\ ${ }^{1}$ Master of Public Health Program and Department of Bioethics, Case Western Reserve University, Cleveland, OH \\ ${ }^{2}$ Department of Bioethics, Case Western Reserve University, Cleveland, $\mathrm{OH}$ \\ Corresponding Author: Michelle A. Gotto, 1220 Huron Road E \#507, Cleveland, OH 44115, (734) 664-1199, michelle.gotto@case.edu \\ Submitted August 20, 2020 Accepted November 29, 2020
}

\section{ABSTRACT}

Background: The United States lags far behind other developed nations in our overall infant mortality rate. Public health researcher Arline Geronimus has described a "weathering" effect of chronic racial stress among Black women that contributes to high rates of preterm birth, the leading cause of infant death. Trusting relationships between clinicians and patients may play a role in reducing infant mortality for Black mothers. Based on a social-ecological model of health care communication around infant mortality, we focus here on doctor-patient communication and correlations between clinicians' understandings of systemic racism and their communication with Black pregnant patients.

Methods: This paper reports the findings from interviews with 5 maternal health clinicians (prior to recruitment being temporarily paused due to COVID-19) practicing at Cuyahoga County hospitals that serve large populations of Black women. Qualitative coding methods based in grounded theory were used to draw out themes from interview transcripts.

Results: Doctor-patient communication was an emergent theme in these interviews. Results suggest an association between clinicians' understanding of the impact of systemic racism and their ability to communicate successfully and form positive bonds with pregnant mothers who are at higher risk of infant mortality.

Conclusion: Acknowledging systemic racism as the cause of poor social determinants of health, which in turn contribute to higher rates of infant mortality, may provide clinicians a pathway to more positive communication and higher levels of trust with their patients, which in turn may play a role in reducing infant mortality in the Black community. Further research should investigate these associations.

Keywords: Infant mortality; Systemic racism; Communication; Clinician-patient relationship; Implicit bias

\section{INTRODUCTION}

The United States began tracking infant death by race in 1850 , when 217 of every 1000 White babies and 340 of every 1000 Black babies did not reach their first birthday. ${ }^{1}$ While the overall US infant mortality rate (IMR, the number of infant deaths per 1000 live births, a leading indicator in the health of a community ${ }^{2}$ ) has dropped dramatically since then, we still lag far behind other wealthy nations (55th overall, with an IMR of 5.833).
These national statistics belie deep geographic, health care access, and, especially, racial disparities: the gap between White and Black IMR is worse now than it was in $1850 .^{1}$ Ohio's 2018 IMR was 6.9, but for Black Ohioans was 13.9.4 And in Cuyahoga County, where the overall 2018 IMR was among Ohio's worst at 8.7, the White IMR was 4.5 (up from 2017 due to the opioid epidemic), and the Black IMR was 14.9.5 While we are seeing progress, Black babies in Cuyahoga County are still dying at 3.3 times the rate of White babies. 
Scholar Dorothy Roberts has argued that the state has historically neglected Black infants via the socioeconomic status (SES) of their mothers through poverty, inadequate housing, poor nutrition, and lack of access to health care. ${ }^{6} \mathrm{Dr}$. Roberts points, for example, to the harsh punishment of pregnant Black women who selfmedicate with illicit drugs versus the "temperate regulation" of pregnant middle-class women who use pharmaceuticals to treat their mental health, despite evidence that use of antidepressants and other prescribed medications during pregnancy may cause subtle neurological problems in newborns. ${ }^{7}$ Such disparities in social treatment contribute to what public health researcher Arline Geronimus has termed "weathering," which she defines as the deterioration of Black women's health due to chronic stress. This stress is caused by the cumulative exposure of socioeconomic disadvantage in a society burdened by structural and systemic racism and has consistently been linked to preterm birth. ${ }^{8}$ Multiple studies have reported associations between hormonal markers of chronic stress and preterm birth, showing disproportionate effects among Black women and women with low SES. ${ }^{9}$ Preterm birth, the leading cause of infant death, has increased 31\% in the United States since 1980, particularly among Black women, and risk identification, early detection, and pharmaceutical interventions have made no impact in reducing its occurrence. ${ }^{9}$

In order to shed light on the marked racial disparities in infant mortality in Cuyahoga County, this study is exploring local clinicians' perceptions of and responses to these issues.

\section{METHODS}

\section{Setting}

The target population was maternal health clinicians from 3 large Cuyahoga County, Ohio, hospitals that serve large numbers of Black women giving birth and large numbers of Medicaid-insured patients.

\section{Design}

This ongoing qualitative research study includes one-on-one semistructured interviews by telephone with maternal health care providers, focus groups with nurses and support staff who work with pregnant patients, and an interactive return of findings to participating hospitals to inform their future practice.

\section{Participants}

This portion of the study was open to physicians of any experience level who work with pregnant patients or patients seeking to become pregnant, at 3 Cuyahoga County hospitals. Before interviews were paused due to the COVID-19 pandemic, our 5 participants included 4 females and 1 male, ranging in experience level from resident to attending physician, with 4 White and 1 Asian participant (Table 1).

\section{Table 1. Participant Demographics}

\begin{tabular}{|cc|}
\hline Gender & \\
Female & 4 \\
Male & 1 \\
\hline Ethnicity & \\
White (Non-Hispanic) & 4 \\
Asian & 1 \\
\hline Clinical Experience & 2 \\
\hline Resident & 1 \\
Fellow & 2 \\
\hline Attending physician & \\
\hline
\end{tabular}

\section{Procedures}

All research methods were approved by the Case Western Reserve University Institutional Review Board (IRB).10 Women's Health departments at 3 Cuyahoga County hospitals agreed to provide access to clinical staff for this study. Maternal health physicians at 3 Cuyahoga County hospitals were contacted via email to participate in this portion of the study; respondents were screened and gave verbal consent by phone, and all were provided with an electronic copy of the IRB-approved study information document. To provide opportunities for in-depth reflection on potentially sensitive topics (particularly infant mortality, systemic racism, and implicit bias in clinical care), individual interviews with maternal health clinicians were conducted. All 60 to 90 minute interviews were conducted via phone by the second author using a guided questionnaire, audio-recorded, and transcribed. Following 5 initial interviews, recruitment was paused due to the COVID-19 pandemic.

\section{Measures/Outcomes}

Participants provided basic demographic information, then were asked open-ended questions about: awareness and understanding of racial disparities in infant mortality at national and local levels (eg, What do you think the most important causes of these health disparities are?); the ways these understandings impacted interactions with Black pregnant patients (eg, How do you see these issues affecting your African American patients here? How have they affected how you interact with your patients?); and awareness and opinions about local efforts to mitigate disparities in infant mortality (including community birth workers and implicit bias training, eg, What do you think about these interventions? What recommendations might you have for your institution?). 


\section{Data Analysis}

Research staff used an approach based in grounded theory,11 with preliminary analyses informing successive interviews and analyses; themes, categories, and theoretical constructs emerged from qualitative coding and analysis. De-identified transcripts were uploaded to Dedoose (www.dedoose.com), a widely-used qualitative analysis software package. The first and third authors created a codebook using 1 interview; both then coded a second interview, resolved discrepancies by consensus, and revised the codebook. To maintain consistency, the first author coded all transcripts, including those used to create the codebook. A matrix was developed to surface major themes from the interviews.

\section{RESULTS}

We interviewed 5 prenatal health care physicians at Cuyahoga County hospitals (Table 1). Analysis of the 5 interviews in this preliminary dataset revealed communication as a consistently emergent theme with implications at multiple levels, although communication was not a primary focus of the interview questions. This primary theme included 4 categories: doctor-patient communication; communication among clinicians; communication between hospitals and outside resources; and communication within the entire care system. For this brief, we focus on the category that was discussed the most by interviewees: doctor-patient communication, which included clinicians' discussions of mistrust from their Black pregnant patients; their awareness of implicit bias in clinical interactions; their understanding of systemic racism and its impact on the social determinants of health; and their understanding of systemic racism as a cause of chronic stress and related symptoms in Black pregnant patients. We found that those clinicians who more readily acknowledged the role of clinicians and health care systems in mistrust and lack of communication also reported more positive interactions with their patients and perceived more trust from those patients.

All 5 interviewees reported perceiving mistrust among their Black patients. Most respondents discussed their understanding that this mistrust comes from a history of Black patients being mistreated in the US medical system. However, their approaches for managing this mistrust varied. Some clinicians' responses alluded to implicit biases, and, regardless of age, some reported feeling challenged in relating to their patients. Three respondents appeared conflicted in accepting that Black women are not heard in the same ways as their White counterparts. For example, Participant 1 noted, "I don't know. It's hard for me to believe that a clinician is going to blow off someone like Serena Williams [who reported that clinicians ignored her life-threatening pulmonary embolism after childbirth]. Do you know what I mean?"

"[There is] a longstanding history of mistrust in the health care system with African-Americans, for good reason given our histo- ry of how they were taken advantage of. I still feel that even today, there's always this level of mistrust when we talk with patients, more so from my African-American patients than any other ethnicity. I'm very conscious when I do counsel them...that I'm explaining as much as I can as clear as I can and offer to talk to their family members or wait for their family to be there." - Participant 5

Two interviewees described a deep awareness of racial disparities in maternal and infant mortality, both nationally and locally, and acknowledged their own biases. They also described independent information seeking on implicit bias and disparities in infant mortality. These 2 also recounted more positive and collaborative encounters with their patients than the other 3 participants:

"I would like to hope that it helps in my counseling. I think it does. It helps me when I make a plan for a patient, and they don't do it exactly as I had envisioned, to take a step back and say, well, maybe why? What other influences are there in your life that are impacting how you're interacting with me and the health care system? What other way can I use to explain what my goal is that maybe makes it easier for you to understand or for us to work on this in a more collaborative approach?...We can find common ground together to work towards our shared goal of healthy mom and baby." - Participant 4

In contrast, the 3 clinicians who reported lower levels of awareness of the causes of infant mortality and were more likely to attribute disparities to lack of access, poverty, or obesity, also reported more difficulty in building trust and establishing rapport with their patients. These clinicians also described frustration with patients they viewed as "noncompliant" or unable to handle basic medical issues. These 3 clinicians reported patients who were skeptical of their recommendations and asked about what they may have seen on the internet or have heard from a family member. They also reported that when these conversations went awry, patients might not return for further appointments. Comparatively, the 2 clinicians who accepted this mistrust as historicallybased and increased their responsiveness to it, reported changing their practice patterns to better accommodate these types of questions and concerns:

"I feel like we just have to make that time. I have a patient in mind right now ... where I'm like, "Do you wanna call your family? I'm happy, whenever they get here, to go over what we've talked about already and the reasons behind that." A lot of patients don't understand what's going on and they just feel like they're in the dark and they're just kind of being ushered around to different things and then that will be a negative memory and a negative experience for them that's going to then impact the rest of their future health care interactions." - Participant 5 
In contrast, other clinicians in our study attributed racial disparities in infant mortality to poor behavior by pregnant women, suggesting that Black women have high-risk pregnancies because they are overweight, have hypertension, or because they are too young, too poor, or unmarried. The clinicians who attributed these disparities to poor behavior by pregnant women were less likely to associate these social determinants of health with systemic racism and were more likely to self-report lower levels of both acceptance in the concept of implicit bias and confidence in implicit bias training.

\section{DISCUSSION}

In these initial interviews with clinicians, an understanding of systemic racism as a cause of both chronic stress and poorer outcomes for Black mothers and babies was associated with an ability to communicate with Black patients in ways that fostered trust. These preliminary associations echo the findings of previous research on doctor-patient communication as a method of building trust with patients, which has found that the physician's approach to the medical encounter matters more than time spent. ${ }^{12}$ Other studies have shown that communication and interpersonal skills, such as an ability to listen and repeat back what the patient has explained, help foster trust. ${ }^{12}$ Clinicians' willingness to ask about health history and psychosocial concerns has been correlated with higher satisfaction among both patients and providers. ${ }^{13}$

This preliminary data suggest that clinician-patient relationships may be influenced by clinicians' understanding of systemic racism and its impact on the social determinants of health. Throughout our interviews, clinicians who showed a higher awareness of systemic racism and accepted systemic racism as a primary factor in the social determinants of health also self-reported more positive and trusting relationships with their patients. In contrast, clinicians who viewed the social determinants of health as being more associated with personal choice and responsibility self-reported more negative and untrusting relationships with their patients.

Though our sample size in this analysis is small, a clear narrative of communication emerged from the interviews. Though communication was not a primary topic of our interview guide, interviewees returned to this theme repeatedly. Our findings suggest that doctor-patient communication may be a vehicle through which clinicians' awareness of systemic racism either improves or hinders their patient relationships, ultimately perhaps affecting their patients' birth outcomes.

We acknowledge that clinicians are often unfairly tasked with solving, or at least mitigating, harms inflicted on Black citizens by centuries of systemic discrimination both outside and inside the medical system. However, one of the tools in reducing infant mortality may be a positive and trusting relationship between the pregnant woman and her clinician, which is made easier when clinicians understand the historical forces facing Black women. This includes an awareness of their own implicit biases, the forces of structural racism in creating the social determinants that may be impacting their compliance, and the cultural norms that influence their behaviors and decisions. Whether that understanding comes from being able to better communicate with their patients-or if being able to better communicate with their patients comes from an initial understanding-is yet to be determined. It may be a mutually-reinforcing cycle, and here is where research with both patients and providers will provide greater understanding.

This study is limited by its small size, which was impacted by the COVID-19 pandemic. No Black clinicians were interviewed, and studies of racial concordance between patients and providers have shown improved outcomes for Black patients.13,14 Further, the research only investigates the point of view of care providers, not patients. When research begins again, additional interviews and focus groups will enhance these initial findings. Further research will be needed to establish any clear correlations between positive communication and positive birth outcomes, both quantitatively and from a patient perspective.

\section{PUBLIC HEALTH IMPLICATIONS}

If additional research supports these preliminary findings, the findings could contribute to guidance for medical schools and hospitals to enhance communication skills among clinicians and other care providers. Trusting and supportive bonds between clinicians and Black pregnant patients could be one aspect of a societal approach to mitigate chronic stress, preterm birth, and infant mortality.

\section{ACKNOWLEDGMENTS}

This project is funded by a faculty research fellowship from the Social Justice Institute at Case Western Reserve University.

\section{REFERENCES}

1. Villarosa L. Why America's black mothers and babies are in a life-ordeath crisis. Published April 11, 2018. Accessed May 10, 2020. https://www.nytimes.com/2018/04/11/magazine/black-mothersbabies-death-maternal-mortality.html

2. Centers for Disease Control and Prevention. Infant Mortality. Published March 27, 2019. Accessed May 20, 2020,

https://www.cdc.gov/reproductivehealth/maternalinfanthealth/ infantmortality.htm

3. Central Intelligence Agency. Country Comparison: Infant Mortality Rate. Published February 01, 2018. Accessed May 20, 2020. https://www.cia.gov/library/publications/resources/the-worldfactbook/fields/354rank.html

4. 2018 Infant Morality Annual Report (Rep.). (2019). https://odh.ohio.gov/wps/wcm/connect/gov/dd1865c0-909c-4378a8e0-61e28364bbae/2018+Ohio+Infant+Mortality+Report.pdf? MOD $=$ AJPERES\&CONVERT_TO $=$ url\&CACHEID $=$ ROOTWORKSPACE.Z1 8_M1HGGIK0N0J000Q09DDDDM3000-dd1865c0-909c-4378-a8e061e28364bbae-n1Z1tQk\#: :text=Section\%201\%3A\%20General\% 20Findings\%20In,compared\%20with\%207.2\%20in\%202017 
5. Health and Human Services Cuyahoga County. The Cuyahoga County Child Fatality Report. Accessed June 9, 2020.

http://protectingourfuture.cuyahogacounty.us/

6. Roberts DE. Killing the Black Body: Race, Reproduction, and the Meaning of Liberty. Vintage Books; 1997;183-184.

7. Roberts DE. Privatization and punishment in the new era of reprogenetics. 2005; Faculty Scholarship at Penn Law. 579:1346-1347.

https://scholarship.law.upenn.edu/faculty_scholarship/579

8. Geronimus AT. Black/white differences in the relationship of maternal age to birthweight: a population-based test of the weathering hypothesis. Soc Sci Med. 1996;42(4):589-597.

https://doi.org/10.1016/0277-9536(95)00159-x

9. Latendresse G. The interaction between chronic stress and pregnancy: preterm birth from a biobehavioral perspective. JMidwifery Womens Health. 2010;54(1):8-17.

https://doi.org/10.1016/j.jmwh.2008.08.001

10. Michie M. (2019) Social Justice Institute, Infant Mortality Research Plan. Case Western Reserve University

11. Charmaz K. Constructing grounded theory: A practical guide through qualitative analysis. Sage Publications. 2006.

12. Dugdale DC, Epstein R, Pantilat SZ. Time and the patient-physician relationship. J Gen Intern Med. 1999;14(S1):S34-40. https://doi.org/10.1046/j.1525-1497.1999.00263.x

13. Traylor AH, Schmittdiel JA, Uratsu CS, Mangione CM, Subramanian U. The predictors of patient-physician race and ethnic concordance: a medical facility fixed-effects approach. Health Serv Res. 2010;45:792805.

https://doi.org/10.1111/j.1475-6773.2010.01086.x

14. Greenwood BN, Hardeman RR, Huang L, Sojourner A. Physicianpatient racial concordance and disparities in birthing mortality for newborns. Proc Natl Acad Sci USA. 2020;117(35): 21194-21200.

https://doi.org/10.1073/pnas.1913405117 